\title{
Highly Efficient Polarized GeS/MoSe 2 van der Waals Heterostructure for Water Splitting from Ultraviolet to Near-Infrared Light
}

\author{
Di Gu, Xiaoma Tao, Hongmei Chen, Weiling Zhu, Yifang Ouyang,* Yong Du, and \\ Qing Peng*
}

A high-efficiency photocatalyst is critical for water splitting by solar light. Herein, via first principles calculations, the 2D polarized $\mathrm{GeS} / \mathrm{MoSe}_{2}$ van der Waals (vdW) heterostructure is proposed as an efficient water redox photocatalyst. The performance of $\mathrm{GeS} / \mathrm{MoSe}_{2}$ heterostructure is better than isolated materials, as the properties of $\mathrm{GeS}$ monolayer and $\mathrm{MoSe}_{2}$ monolayer are complementary by forming vdW heterostructure. $\mathrm{GeS} / \mathrm{MoSe}_{2}$ heterostructure possesses suitable bandgap, dipole-induced internal electric field, and excellent solar absorption performance. The band alignments of $\mathrm{GeS} / \mathrm{MoSe}_{2}$ heterostructure are suitable compared with the redox potential of water. It is feasible to tune the optoelectronic properties and enhance photocatalytic activity of $\mathrm{GeS} / \mathrm{MoSe}_{2}$ heterostructure via strain engineering. Biaxial compressive strain range from $-2 \%$ to $-3 \%$ induces the direct bandgap characteristic in $\mathrm{GeS} / \mathrm{MoSe}_{2}$ heterostructure. The results suggest that $2 \mathrm{D}$ polarized $\mathrm{GeS} / \mathrm{MoSe}_{2}$ vdW heterostructure is a potential novel high-efficiency photocatalyst for water splitting under a wide range of spectra from ultraviolet to near infrared.

electron-hole pairs separate and transfer to the surface of photocatalysts, and the $\mathrm{H}_{2}\left(\mathrm{O}_{2}\right)$ are generated by the way of hydrogen evolution reaction (oxygen evolution reaction) on the surface of photocatalysts, respectively. ${ }^{[4,5]}$ Therefore, effective solar light harvesting and carrier separation are keys to enhance the efficiency of the photocatalytic water-splitting process. Since Fujishima and Honda ${ }^{[6]}$ reported photocatalytic water splitting in 1972 first, bulk materials, such as $\mathrm{TiO}_{2},{ }^{[7-10]} \mathrm{ZnO},{ }^{[11,12]}$ $\mathrm{CdS},{ }^{[13-15]}$ and $\mathrm{SrTiO}_{3},{ }^{[16,17]}$ have been extensively investigated as potential photocatalysts. However, the conversion efficiency of these bulk materials is still low and unsatisfactory due to the large bandgap, low solar light absorption, low carrier mobility, and high carrier recombination rate. ${ }^{[5,18]}$ Compared with the traditional bulk materials, a series of studies

Harvesting solar light is an ultimate solution to energy crisis. Photocatalytic water-splitting technology, in which process solar light, water, and catalyst are adopted to generate $\mathrm{O}_{2}$ and $\mathrm{H}_{2}$ without polluting the environment, has been considered as an efficient and feasible approach to solve the serious energy and environmental problems. ${ }^{[1-3]}$ There are three major processes in photocatalytic water splitting, as the photogenerated

D. Gu, Prof. X. Tao, Prof. H. Chen, Prof. Y. Ouyang

School of Physical Science and Technology

Guangxi University

Nanning 530004, P. R. China

E-mail: ouyangyf@gxu.edu.cn

D. Gu

School of Chemistry and Chemical Engineering

Guangxi University

Nanning 530004, P. R. China

D. Gu, Prof. W. Zhu

Department of Physics

School of Science

Guangdong University of Petrochemical Technology

Maoming, Guangdong 525000, P. R. China

The ORCID identification number(s) for the author(s) of this article can be found under https://doi.org/10.1002/pssr.201900582. reported that 2D materials have distinctive advantageous properties for photocatalytic water splitting, such as a large specific surface area, short distance for charge carrier diffusion, high carrier mobility, low carrier recombination rate, strong visible light absorption, and novel electronic properties, ${ }^{[19-22]}$ due to the decrease in the dimension of materials and quantum confinement.

DOI: 10.1002/pssr.201900582 
Recent researches have manifested that some 2D polarized materials with dipole moment induce vertical intrinsic electric field throughout the whole material, which implies promising excellent applications in photocatalytic water splitting. ${ }^{[23-26]}$ The induced vertical intrinsic electric field not only efficiently separates carriers from interior to surface, but also effectively reduces the photocatalyst's bandgap required for water splitting, leading to a widened solar light absorption region. ${ }^{[27]}$ Therefore, some 2D polarized materials have been theoretically and experimentally proved to be potential photocatalysts for water splitting. For example, Yang and coworkers ${ }^{[28]}$ reported that the intrinsic electric fields in experimentally attainable $2 \mathrm{D} \mathrm{In}_{2} \mathrm{Te}_{3}$ enhanced the performance for photocatalytic water splitting and the theoretical efficiency of solar to hydrogen using the full solar spectrum and even achieved $32.1 \%$. Liu and coworkers ${ }^{[22]}$ experimentally confirmed that $2 \mathrm{D}$ material $\left(\mathrm{WS}_{2}\right.$ nanosheet) with dipole possessed full solar light spectrum photodegradation activity and high photocatalytic efficiency. Recently synthesized 2D Janus MXY ( $\mathrm{M}=\mathrm{Mo}, \mathrm{X} / \mathrm{Y}=\mathrm{S}, \mathrm{Se}, \mathrm{Te})$ are also potential photocatalysts for water splitting with a low carrier recombination rate due to structural symmetry breaking and induced internal electric field. ${ }^{[25,29-31]}$ Moreover, the switch of the dipole moment controlled by the stacking order indicates that asymmetric 2D Janus MXY $(\mathrm{M}=\mathrm{Mo}, \mathrm{W}, \mathrm{X} / \mathrm{Y}=\mathrm{S}, \mathrm{Se}, \mathrm{Te})$ is responsive to light with a wide-range spectrum from infrared (IR) to ultraviolet (UV). ${ }^{[24]}$ Our previous study ${ }^{[32]}$ has demonstrated that the blue phosphorene-phase monolayer $\mathrm{GeS}$ and GeSe possesses unique photocatalytic properties for water splitting, and strain engineering can enhance photocatalytic activity under visible light due to the internal electric field induced by the dipole moment from the Ge atomic surface to $\mathrm{S} / \mathrm{Se}$ atomic surface.

Although the 2D polarized materials possess promising potential applications in photocatalysts of water splitting, however, there's definitely room for improvement, considering the properties are limited by single material system. Recently, many studies have shown that forming van der Waals (vdW) heterostructures based on different monolayer 2D materials is an effective and facile way to combine the advantages of different 2D materials. ${ }^{[33-36]}$ For example, Deng et al. reported that a photodetector based on $\mathrm{MoS}_{2}$ /black phosphorus vdW heterostructure showed a photodetection responsivity of $418 \mathrm{~mA} \mathrm{~W}^{-1}$, which was much higher than the value of $4.8 \mathrm{~mA} \mathrm{~W}^{-1}$ for the single black phosphorus phototransistors. ${ }^{[34]}$ Moreover, many recent studies have shown that constructing photocatalysts based on vdW heterostructures, such as GeS/WS ${ }_{2},{ }^{[37]} \mathrm{GaS} / \mathrm{g}-\mathrm{C}_{3} \mathrm{~N}_{4},{ }^{\left[{ }^{38]}\right.} \mathrm{MoS}_{2} /$

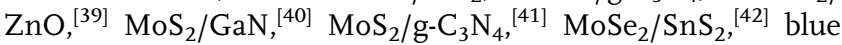
$\mathrm{P} / \mathrm{Mg}(\mathrm{OH})_{2},{ }^{\left[{ }^{[4]}\right.}$ blue P/AlN, ${ }^{[44]}$ blue P/BSe, ${ }^{[45]} \mathrm{BCN} / \mathrm{C}_{2} \mathrm{~N}^{[46]}$ and $\alpha-\mathrm{Fe}_{2} \mathrm{O}_{3} / g-\mathrm{C}_{3} \mathrm{~N}_{4},{ }^{[47]}$ can enhance the photocatalytic efficiency for water splitting. ${ }^{[5,18,48,49]}$ Considering the excellent advantage properties of the $2 \mathrm{D}$ polarized materials and the heterostructures for water splitting, exploring heterostructures based on the 2D polarized materials is a potential effective way to enhance the photocatalytic performance of the water-splitting process. Therefore, it is thus important and interesting to design and investigate the vdW heterostructures based on the 2D polarized materials.

Here, we design and investigate the vdW heterostructures based on the $2 \mathrm{D}$ polarized materials, using $\mathrm{GeS} / \mathrm{MoSe}_{2}$ as the demonstration. GeS monolayer shared a similar hexagonal crystal structure and nearly identical lattice constants with $\mathrm{MoSe}_{2}$ monolayer, which possesses a sizable direct bandgap, advantageous optoelectronic properties, and distinguished mechanical properties. We expected that the advantageous properties of $\mathrm{GeS}$ and $\mathrm{MoSe}_{2}$ were well preserved by forming the $\mathrm{GeS} / \mathrm{MoSe}_{2}$ vdW heterostructure. The structural stability, binding energy, internal electric field, optoelectronic properties, and photocatalytic activity of the $\mathrm{GeS} / \mathrm{MoSe}_{2}$ heterostructure were systematically investigated via first principles calculations. The effect of biaxial strain on the properties of $\mathrm{GeS} / \mathrm{MoSe}_{2} \mathrm{vdW}$ heterostructure was also discussed. The results suggest that the $2 \mathrm{D}$ polarized material-based $\mathrm{GeS} / \mathrm{MoSe}_{2} \mathrm{vdW}$ heterostructure is a potential novel high-efficiency photocatalyst for water splitting under a wide range from UV to near-IR light.

The optimized lattice constants of $\mathrm{GeS}$ and $\mathrm{MoSe}_{2}$ monolayers are 3.495 and $3.319 \AA$, respectively, agreeing well with previous studies. ${ }^{[32,50-52]}$ The lattice constants' difference between GeS and $\mathrm{MoSe}_{2}$ monolayer, or the lattice mismatch, is about $2.4 \%$ which is in an acceptable range ${ }^{[53,54]}$ and good for constructing heterostructures along $z$ direction. The stacking configuration of the polarized material-based heterostructure is more complex than that based on the nonpolarized materials, because the GeS monolayer possesses a dipole-induced internal electric field which is from the Ge atomic surface to $\mathrm{S}$ atomic surface, indicating that both the Ge atomic surface and $\mathrm{S}$ atomic surface should be considered to contact with the $\mathrm{MoSe}_{2}$ monolayer when stacking the $\mathrm{GeS} / \mathrm{MoSe}_{2}$ heterostructures. As shown in Figure S1, Supporting Information, 12 stacking structures of GeS/ $\mathrm{MoSe}_{2}$ heterostructures are considered, namely A1-, A2-, A3-, B4-, B5-, B6-, C7-, C8-, C9-, D10-, D11-, and D12- stacking, respectively. In the A1-stacking, the $\mathrm{Ge}$ atomic surface approaches the $\mathrm{MoSe}_{2}$ monolayer and the GeS monolayer is directly stacked on the $\mathrm{MoSe}_{2}$ of bottom layer, in which $\mathrm{Ge}$ atoms just locate above Mo atoms, and $\mathrm{S}$ atoms are just situated in the center of hexagon of the $\mathrm{MoSe}_{2}$ monolayer. Moreover, the A2- and A3-stacking can be obtained as the $\mathrm{MoSe}_{2}$ monolayer is translated along the vectors of $(b-a)$ direction with the distance of about $1 / 3$ or $2 / 3$ length of the unit cell, respectively, whereas the GeS monolayer is unchanged. Comparing with the A-combination, the direction of $\mathrm{MoSe}_{2}$ monolayer in the B-combination is along the opposite. The B1-, B2-, and B3-stacking can be obtained as that the bottom-layer $\mathrm{MoSe}_{2}$ is rotated $180^{\circ}$ along the $z$ direction from A3-, A1-, and A2-stacking, respectively. The C-combination (D-combination) is similar to the A-combination (B-combination), respectively, whereas the $\mathrm{MoSe}_{2}$ monolayer is directly stacked on the $\mathrm{GeS}$ of the bottom layer, as the $\mathrm{S}$ atomic surface is chosen to approach the $\mathrm{MoSe}_{2}$ monolayer.

To obtain a stable atomic configuration, all 12 stacking structures are fully relaxed, and the total energy of each stacking is calculated and counted. As shown in Figure S2, Supporting Information, the B5-stacking gets the minimum value among the 12 stacking structures, indicating that B5-stacking is the most stable structure. Therefore, the B5-stacking (Figure 1a) was chosen as the optimum representative stacking to show the properties of the $\mathrm{GeS} / \mathrm{MoSe}_{2}$ vdW heterostructure in the following paragraphs. The energy as a function of lattice constant is shown in the Figure S3, Supporting Information, the result indicates that the optimum lattice constant of $\mathrm{GeS} / \mathrm{MoSe}_{2}$ heterostructure is about $3.405 \AA$. To analyze the thermodynamic stability, the 
(a)
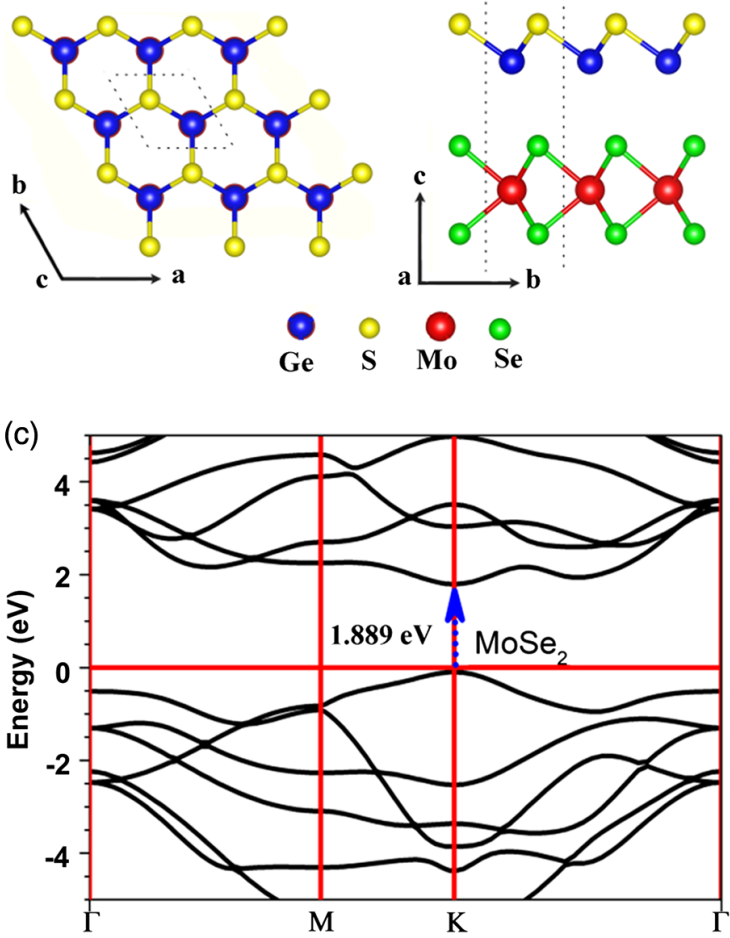

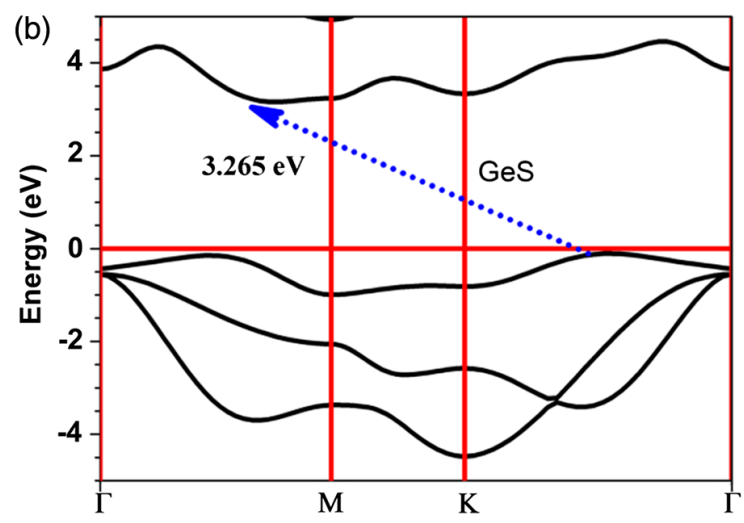

(d)

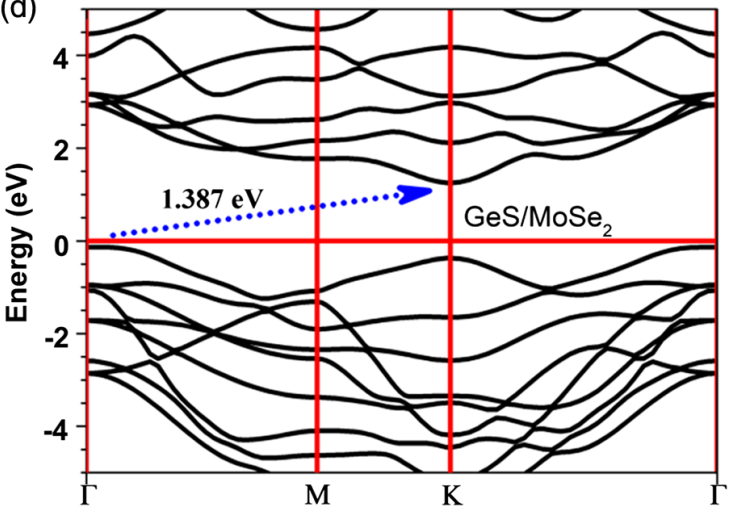

Figure 1. a) Top and side views of optimum stacking order of the GeS/MoSe 2 heterostructure. The band structures (HSE06) of b) monolayer GeS, c) monolayer $\mathrm{MoSe}_{2}$, and d) $\mathrm{GeS} / \mathrm{MoSe}_{2}$ heterostructure.

binding energy and phonon spectrum of $\mathrm{GeS} / \mathrm{MoSe}_{2}$ heterostructure were calculated. The binding energy $E_{\mathrm{d}}$ is calculated using the following definition

$E_{\mathrm{d}}=\frac{E_{\mathrm{h}}-E_{\mathrm{GeS}}-E_{\mathrm{MoSe}_{2}}}{A}$

where $E_{\mathrm{h}}, E_{\mathrm{GeS}}$, and $E_{\mathrm{MoSe}}$ represent the total energy of the $\mathrm{GeS} / \mathrm{MoSe}_{2}$ heterostructure, isolated GeS monolayer, and isolated $\mathrm{MoSe}_{2}$ monolayer, respectively, and $A$ is the area of $\mathrm{GeS} / \mathrm{MoSe}_{2}$ heterostructure. A more negative binding energy indicates a more thermodynamically stable structure. The binding energy of $\mathrm{GeS} / \mathrm{MoSe}_{2}$ heterostructure is about $-23.76 \mathrm{meV} \AA^{-2}$, which is close to that of other vdW heterostructures, ${ }^{[39,43,55,56]}$ indicating that $\mathrm{GeS} / \mathrm{MoSe}_{2}$ heterostructure is thermodynamically stable. As shown in Figure S4 and S5, Supporting Information, there is no imaginary vibration mode in the phonon spectrum of GeS/MoSe $e_{2}$ heterostructure, confirming the dynamical stability of this structure.

The band structures of the isolated $\mathrm{GeS}, \mathrm{MoSe}_{2}$ monolayer, and the $\mathrm{GeS} / \mathrm{MoSe}_{2}$ heterostructure were calculated based on the HSE06 method. As shown in Figure 1b, the isolated GeS monolayer is an indirect semiconductor with a large bandgap, and the conduction band minimum (CBM) appears between the $\Gamma$ and $M$ points, whereas the valence band maximum (VBM) appears between the $K$ and $\Gamma$ points. The GeS monolayer has been reported as a potential promising photocatalyst for water splitting, due to a dipole moment-induced vertical intrinsic electric field in the monolayer, which is helpful for separating the carriers from the interior to the surface. However, the value of the bandgap of the GeS monolayer is about $3.265 \mathrm{eV}$, similar to that of the conventional bulk material photocatalysts ${ }^{[8,12]}$ such as $\mathrm{TiO}_{2}$, indicating that only violet light, just about $5 \%$ of the total energy of solar light, can be harvested, which greatly limits its applications.

The isolated $\mathrm{MoSe}_{2}$ monolayer is a direct semiconductor with a bandgap of about $1.889 \mathrm{eV}$, and both the CBM and VBM locate at the $K$ point (Figure 1c), in good agreement with previous results reported. ${ }^{[51,52]}$ The GeS/MoSe 2 heterostructure is a semiconductor with an indirect bandgap, and the CBM and VBM locate at $\Gamma$ point and $K$ point, respectively (Figure $1 \mathrm{~d}$ ). The band structures of $\mathrm{GeS} / \mathrm{MoSe}_{2}$ heterostructure preserve both the properties of isolated $\mathrm{GeS}$ and $\mathrm{MoSe}_{2}$ monolayers. The bandgap of the $\mathrm{GeS} / \mathrm{MoSe}_{2}$ heterostructure is about $1.387 \mathrm{eV}$, which is smaller than those of the isolated $\mathrm{GeS}$ and $\mathrm{MoSe}_{2}$ monolayer due to the formation vdW heterostructure. As a result, GeS/MoSe 2 heterostructure can harvest a broader light spectrum range from UV to visible solar light; compared with that only UV light is harvested by the isolated GeS monolayer. Therefore, formatting the $\mathrm{GeS} / \mathrm{MoSe}_{2}$ heterostructure is an efficient way to work out the problem that the isolated GeS monolayer faced and enhance solar light harvesting effectively.

What is more, the band structures for monolayer GeS, monolayer $\mathrm{MoSe}_{2}$, and $\mathrm{GeS} / \mathrm{MoSe}_{2}$ heterostructure including 
the effect of spin-orbit coupling (SOC) are shown in Figure S6, Supporting Information. It is clearly shown that there is no significant SOC effect on the monolayer GeS, whereas there is an SOC effect on $\mathrm{MoSe}_{2}$ and $\mathrm{GeS} / \mathrm{MoSe}_{2}$ heterostructure. It can be found that SOC slightly influences bandgap values while maintaining the band-structure characteristics and shapes. To shed light on the fundamental mechanism of the orbital reconfiguration of the $\mathrm{GeS} / \mathrm{MoSe}_{2}$ heterostructure, the partial density of states (PDOS) and the band-decomposed charge density of the VBM and CBM are shown in Figure S7, Supporting Information. It is shown that it is $p$ orbitals of $\mathrm{Ge}, \mathrm{S}$, and Se, and d orbitals of Mo that contributed mainly to the VBM below the Fermi level, whereas p orbitals of Se and $\mathrm{d}$ orbitals of Mo contributed mainly to the CBM above the Fermi level.

Due to charge redistribution, the 2D polarized materials possess an intrinsic dipole moment $P$ through the materials. This intrinsic dipole moment $P$ induces a surface potential difference $(\Delta \Phi)$ between the vacuum levels of the top and bottom surface. As shown in Figure $2 \mathrm{a}-\mathrm{c}$, for the isolated $\mathrm{GeS}$ monolayer, there is $\Delta \Phi$ of about $0.793 \mathrm{eV}$ between the bottom surface (Ge atomic side) and top surface ( $\mathrm{S}$ atomic side) for the monolayer $\mathrm{GeS}$. The direction pointing in the internal electric field $\left(E_{\text {eff }}\right)$ is from the bottom surface to top surface. As a contrast, $\Delta \Phi$ is zero for $\mathrm{MoSe}_{2}$ monolayer, as the $\mathrm{MoSe}_{2}$ monolayer is a
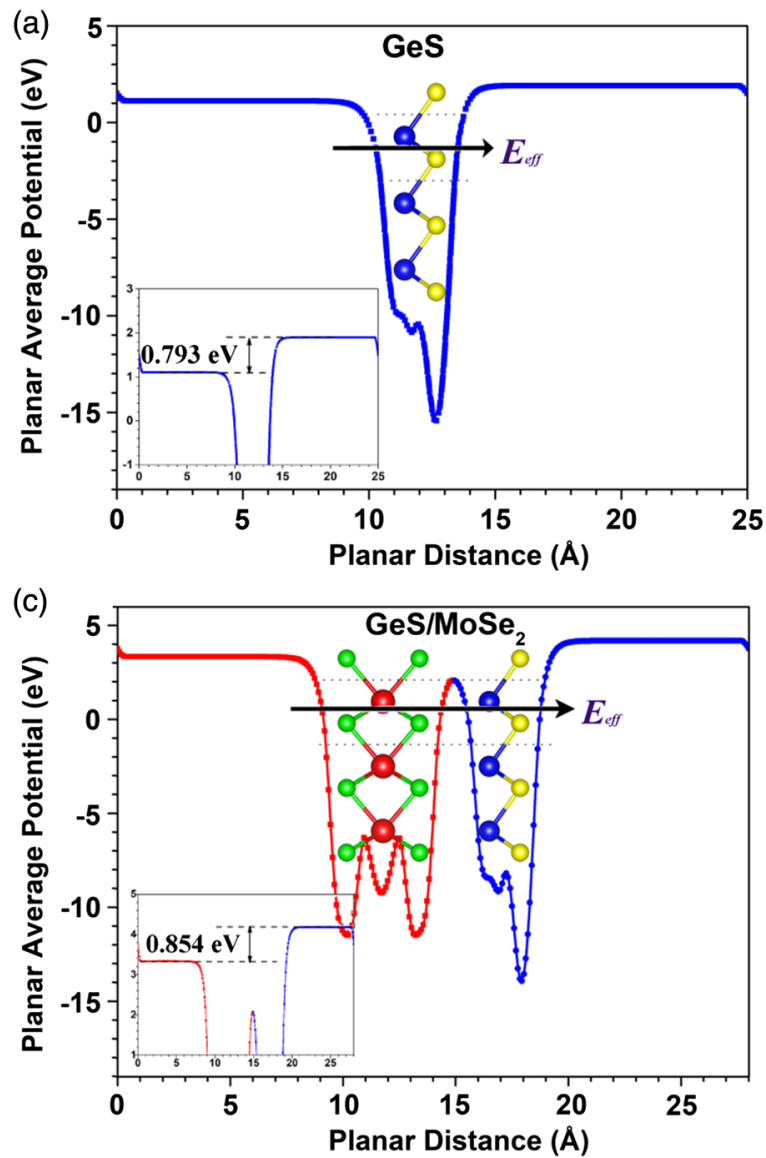

nonpolarized 2D material with the same element on both sides. Importantly, for the $\mathrm{GeS} / \mathrm{MoSe}_{2}$ heterostructure, the surface potential difference $\Delta \Phi$ is considerable. The vacuum level of the bottom surface ( $\mathrm{MoSe}_{2}$ monolayer side) is lower than that of the top surface ( $\mathrm{GeS}$ monolayer side). As a consequence, there is an intrinsic electric field in the $\mathrm{GeS} / \mathrm{MoSe}_{2}$ heterostructure. The direction is from the bottom surface to the top surface, consistent with that of the isolated $\mathrm{GeS}$ monolayer. Our result confirms that the $\mathrm{GeS} / \mathrm{MoSe}_{2}$ heterostructure preserves the properties of the dipole-induced internal electric field, which is a distinct and advantageous property in the 2D polarized materials. Furthermore, the value of $\Delta \Phi$ of the GeS/ $\mathrm{MoSe}_{2}$ heterostructure is about $0.854 \mathrm{eV}$, larger than that of the isolated $\mathrm{GeS}$ monolayer, suggesting that the band bending across the junction surface can induce this surface potential difference.

To unveil the mechanism of the dipole-induced internal electric field of the $\mathrm{GeS} / \mathrm{MoSe}_{2}$ heterostructure, the charge density difference and the plane-average charge density difference along the $z$ direction of $\mathrm{GeS} / \mathrm{MoSe}_{2}$ heterostructure were calculated and shown in Figure $2 \mathrm{~d}$,e, respectively. The charge density difference $\Delta \rho$ can be calculated according to the following equation

$$
\Delta \rho=\rho_{\text {heterostructure }}-\rho_{\mathrm{GeS}}-\rho_{\mathrm{MoSe}_{2}}
$$
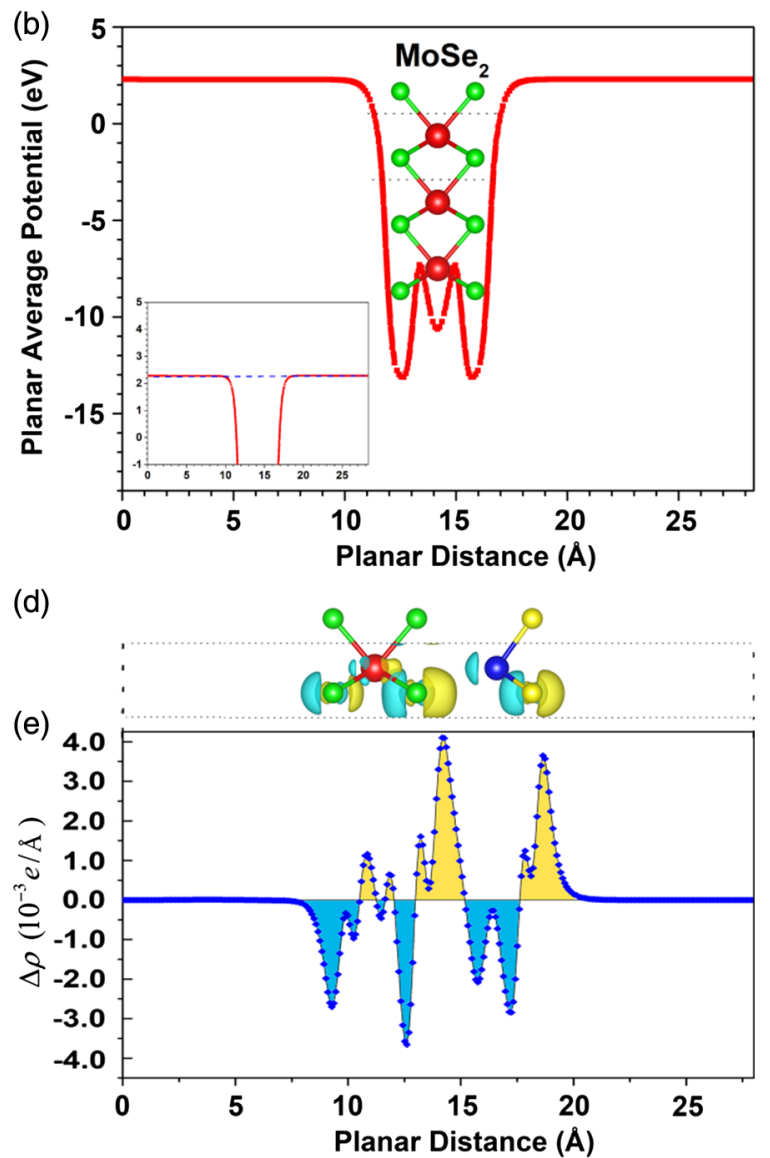

Figure 2. The planar average potential of a) monolayer $\mathrm{GeS}$, b) monolayer $\mathrm{MoSe}_{2}$, and c) $\mathrm{GeS} / \mathrm{MoSe}_{2}$ heterostructure. d) The charge density difference and e) the plane-average charge density difference along the $z$ direction of $\mathrm{GeS} / \mathrm{MoSe}_{2}$ heterostructure. 
in which $\rho_{\text {heterostructure }} \rho_{\mathrm{GeS}}$, and $\rho_{\mathrm{MoSe}}$ are the charge densities of the $\mathrm{GeS} / \mathrm{MoSe}_{2}$ heterostructure, isolated $\mathrm{GeS}$ monolayer, and isolated $\mathrm{MoSe}_{2}$ monolayer with the same in-plane lattice constant, respectively. Moreover, the plane-average charge density difference along the $z$ direction $\Delta \rho(z)$ can be obtained by integrating the in-plane charge density difference $\Delta \rho$ according to the following equation

$$
\begin{aligned}
\Delta \rho(z)= & \int \rho_{\text {heterostructure }}(x, y, z) \mathrm{d} x \mathrm{~d} \gamma-\int \rho_{\mathrm{GeS}}(x, y, z) \mathrm{d} x \mathrm{~d} y \\
& -\int \rho_{\mathrm{MoSe}_{2}}(x, y, z) \mathrm{d} x \mathrm{~d} y
\end{aligned}
$$

Herein, yellow and blue isosurfaces correspond to charge accumulation and depletion, respectively. As shown in Figure $2 \mathrm{~d}$,e, the Ge atom denotes electrons to the $\mathrm{S}$ atom, and the holes remained in the $\mathrm{Ge}$ atom; therefore, the $\mathrm{Ge}$ atom gathers positive charges and the $\mathrm{S}$ atom gathers negative charges, confirming that the dipole-induced internal electric field is from Ge atomic side to $\mathrm{S}$ atomic side which is mentioned earlier in Figure 2a.

Moreover, the nonpolarized $\mathrm{MoSe}_{2}$ monolayer is unavoidably influenced by the polarized $\mathrm{GeS}$ monolayer, after forming the vdW GeS/MoSe 2 heterostructure. For the $\mathrm{MoSe}_{2}$ layer, the charge redistribution behavior is regular. The electrons of each atom of the $\mathrm{MoSe}_{2}$ layer transfer from the further section to the nearer section which approaches the GeS layer, leading to the polarization of electrons and the formation of the internal electric field induced by dipole moment. This means the dipole-induced internal electric field appears in both the GeS layer and $\mathrm{MoSe}_{2}$ layer of the $\mathrm{GeS} / \mathrm{MoSe}_{2}$ heterostructure. The direction pointing in the $\mathrm{MoSe}_{2}$ layer is similar to that of the GeS layer. The dipoleinduced internal electric field of the $\mathrm{GeS} / \mathrm{MoSe}_{2}$ heterostructure is from the bottom surface to the top surface, which is consistent well with the result analyzed from the surface potential difference.

The 2D polarized material-based $\mathrm{GeS} / \mathrm{MoSe}_{2}$ heterostructure preserves the advantageous properties, such as the unique dipole-induced internal electric field, as same as those of the 2D polarized GeS monolayer. The dipole-induced internal electric field $E_{\text {eff }}$ of $\mathrm{GeS} / \mathrm{MoSe}_{2}$ heterostructure is helpful for the efficiency of separating carriers, reducing effectively the probability of recombination of the photogenerated electron-hole pairs and ensuring the photocatalytic activity.

The solar light harvest is the premier step in the photocatalytic water-splitting process, of which, the efficiency is a crucial factor. A desirable photocatalyst should possess not only a strong solar light absorption ability, but also a wide solar light adsorption range, especially visible light and IR light. To elucidate the absorption performance, the absorption spectra were obtained by calculating the dielectric function according the following equation

$\alpha(\omega)=\sqrt{2} \omega\left(\sqrt{\left(\varepsilon_{1}(\omega)\right)^{2}+\left(\varepsilon_{2}(\omega)\right)^{2}}-\varepsilon_{1}(\omega)\right)^{1 / 2}$

Here, the absorption coefficient is represented by $\alpha(\omega)$, the real and imaginary parts of the frequency-dependent complex dielectric function correspond to $\varepsilon_{1}(\omega)$ and $\varepsilon_{2}(\omega)$, respectively. As shown in Figure 3a, the optical absorption coefficients as a function of wavelength for the isolated $\mathrm{GeS}$ monolayer, isolated $\mathrm{MoSe}_{2}$ monolayer, and the $\mathrm{GeS} / \mathrm{MoSe}_{2}$ heterostructure were calculated. The isolated GeS monolayer only absorbs UV light, for which the proportion is just only about $5 \%$ of total solar light. The visible and IR light that accounts for the most proportion of solar light cannot be utilized due to the large bandgap of about $3.265 \mathrm{eV}$ for $\mathrm{GeS}$ monolayer, leading to low solar absorption performance. In contrast, the isolated $\mathrm{MoSe}_{2}$ monolayer possesses excellent solar absorption performance, such as wide solar light adsorption ranging from the UV light to the visible light, strong solar light-absorption ability, and the absorption coefficients near $10^{6} \mathrm{~cm}^{-1}$, agreeing well with the precious reported. ${ }^{[57]}$ More importantly, vdW heterostructures can absorb solar light more efficiently than the isolated materials, ${ }^{[57-59]}$ as shown in the $\mathrm{GeS} / \mathrm{MoSe}_{2}$ heterostructure in this study. The excellent solar absorption performance of isolated $\mathrm{MoSe}_{2}$ monolayer can be preserved in the $\mathrm{GeS} / \mathrm{MoSe}_{2}$ heterostructure. The absorption coefficient of the $\mathrm{GeS} / \mathrm{MoSe}_{2}$ heterostructure is slightly larger than that of the isolated $\mathrm{MoSe}_{2}$ monolayer. In addition, as shown in Figure $3 \mathrm{a}$ and Figure S8, Supporting Information, the
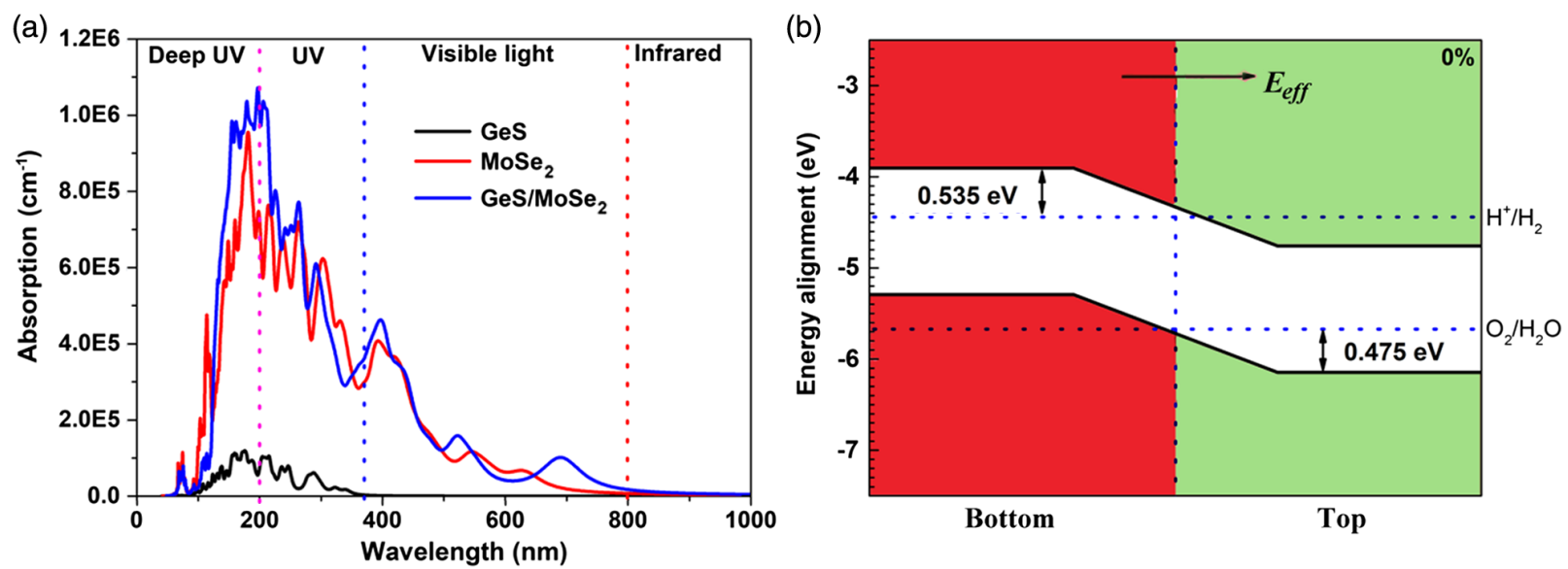

Figure 3. a) Absorption coefficients of monolayer $\mathrm{GeS}$, monolayer $\mathrm{MoSe}_{2}$, and $\mathrm{GeS} / \mathrm{MoSe}_{2}$ heterostructure. b) The energy alignment of GeS/MoSe heterostructure. 
absorption edge of the $\mathrm{GeS} / \mathrm{MoSe}_{2}$ heterostructure has redshifted to near-IR light, due to the bandgap reduction to about $1.387 \mathrm{eV}$ after formatting vdW heterostructure. The results of absorption spectrum suggest that $\mathrm{GeS} / \mathrm{MoSe}_{2}$ heterostructure possesses excellent solar absorption performance, which is contributed mainly by $\mathrm{MoSe}_{2}$ monolayers. A wide light spectrum ranging from UV to near-IR light can be utilized with a high absorption coefficient, which is useful to enhance the solar energy conversion performance for water splitting.

To examine the possible applications of $\mathrm{GeS} / \mathrm{MoSe}_{2}$ heterostructure in photocatalytic water splitting, the band alignments of the GeS/MoSe 2 heterostructure were compared with the redox potential of water. The dipole moment-induced vertical intrinsic electric field in the 2D polarized materials will induce the energy level band, ${ }^{[27]}$ reducing the photocatalyst's bandgap requirement for water splitting. The energy alignment of $\mathrm{GeS} / \mathrm{MoSe}_{2}$ heterostructure was surveyed according to the methods used in the polarized materials, as previous papers reported, ${ }^{[23,25-27,32]}$ in which the VBM was assumed to be equal to the work function according the following equation

$E_{\mathrm{VBM}}=\varphi(\infty)-E_{\mathrm{F}}$

where $\varphi(\infty)$ is the electrostatic potential in vacuum and $E_{\mathrm{F}}$ is the Fermi energy level, and the CBM was calculated according the following equation

$E_{\mathrm{CBM}}=E_{\mathrm{VBM}}+E_{\text {gap }}$

where $E_{\text {gap }}$ is the value of the bandgap (HSE06).

As shown in Figure 3b, the dipole moment-induced vertical intrinsic electric field is from the bottom surface to the top surface, which is helpful to separate photogenerated electrons and holes effectively. Therefore, the electrons transfer from interior to the bottom surface, whereas the holes transfer from interior to the top surface, respectively. On the top side, the VBM is lower than the oxidation potential of $\mathrm{O}_{2} / \mathrm{H}_{2} \mathrm{O}(-5.67 \mathrm{eV})$, and the energy difference $\left(\Delta E_{\mathrm{v}}\right)$ between the VBM level and the oxidation potential level is $0.475 \mathrm{eV}$, which is suitable to drive holes to oxidize $\mathrm{H}_{2} \mathrm{O}$ to $\mathrm{O}_{2}$

$4 \mathrm{~h}^{+}+2 \mathrm{H}_{2} \mathrm{O} \rightarrow \mathrm{O}_{2}+4 \mathrm{H}^{+}$

On the bottom side, the CBM is higher than the reduction potential of $\mathrm{H}^{+} / \mathrm{H}_{2}(-4.44 \mathrm{eV})$, and the energy difference $\left(\Delta E_{\mathrm{c}}\right)$ between the CBM level and the reduction potential level of $\mathrm{H}^{+} / \mathrm{H}_{2}$ is $0.535 \mathrm{eV}$, which is suitable to urge electrons to catalyze $\mathrm{H}_{2} \mathrm{O}$ to $\mathrm{H}_{2}$

$4 \mathrm{e}^{-}+4 \mathrm{H}_{2} \mathrm{O} \rightarrow 2 \mathrm{H}_{2}+4 \mathrm{OH}^{-}$

In addition, the value of the $\Delta E_{\mathrm{v}}$ is similar to that of $\Delta E_{\mathrm{c}}$, indicating a harmonious ability to produce hydrogen and oxygen. In general, the results suggest that $\mathrm{GeS} / \mathrm{MoSe}_{2}$ vdW heterostructure is a potential high-efficiency photocatalyst for water splitting, as it possesses a dipole moment-induced vertical intrinsic electric field and a suitable band alignment under a wide range from UV to near-IR light.

Strain engineering is an effective and convenient strategy to tune the optoelectronic performance of $2 \mathrm{D}$ materials. ${ }^{[51,60-64]}$
Herein, the electronic, optical, and photocatalytic properties of the $\mathrm{GeS} / \mathrm{MoSe}_{2}$ heterostructure under external biaxial strain were systematically investigated. Compressing and stretching are represented by symbols "-" and "+", respectively. The band structures (HSE06) of $\mathrm{GeS} / \mathrm{MoSe}_{2}$ heterostructure at various biaxial strains are shown in Figure $4 \mathrm{a}-\mathrm{c}$, and the band gap as a function of biaxial strain ranging from 0 to $\pm 10 \%$ is shown in Figure 4d. The magnitude of the bandgap for the $\mathrm{GeS} /$ $\mathrm{MoSe}_{2}$ heterostructure can be tuned continuously by biaxial strain. For tensile strain, the bandgap monotony decreased from 1.387 to $0.002 \mathrm{eV}$ when the biaxial strain increased from 0 to $+10 \%$, indicating that more visible light and IR light can be harvested by $\mathrm{GeS} / \mathrm{MoSe}_{2}$ heterostructure, which is also confirmed by the absorption spectrum shown in Figure S9, Supporting Information. For compressive strain, the bandgap increases to the maximum of $2.064 \mathrm{eV}$, when the biaxial strain decreased from 0 to $-4 \%$. The bandgap decreased as the biaxial strain decreases from $-4 \%$ to $-10 \%$.

Under the compressive strain range from $-2 \%$ to $-3 \%, \mathrm{GeS} /$ $\mathrm{MoSe}_{2}$ heterostructure possesses a direct bandgap character and the VBM and CBM both appear in the $K$ point. More details are shown in Figure $4 \mathrm{~b}$ and Figure S10, Supporting Information, the CBMs and VBMs of $\mathrm{GeS} / \mathrm{MoSe}_{2}$ heterostructure at $-2 \%$ and $-3 \%$ biaxial strain are similar to that of monolayer $\mathrm{MoSe}_{2}$. The lattice constants and bandgap of $\mathrm{GeS} / \mathrm{MoSe}_{2}$ heterostructure at $-2 \%(-3 \%)$ biaxial strain are $3.336 \AA$ (3.302 $\AA$ ) and $1.845 \mathrm{eV}$ $(1.968 \mathrm{eV})$, which are just close to $3.319 \AA$ and $1.889 \mathrm{eV}$ of monolayer $\mathrm{MoSe}_{2}$, respectively, indicating that the direct bandgap character of $\mathrm{GeS} / \mathrm{MoSe}_{2}$ heterostructure is mainly contributed by the monolayer $\mathrm{MoSe}_{2}$. Therefore, it is feasible to tune the properties and enhance photocatalytic activity of $\mathrm{GeS} / \mathrm{MoSe}_{2}$ heterostructure via strain engineering.

To make further inquiry into the possible applications in photocatalytic water splitting, the band alignments of the $\mathrm{GeS} / \mathrm{MoSe}_{2}$ heterostructure under biaxial strain are shown in Figure 5 and Figure S11, Supporting Information. As shown in Figure $5 a-c$, when the biaxial strain is $+2 \%,-2 \%$, and $-6 \%$, the $\mathrm{GeS} / \mathrm{MoSe}_{2}$ heterostructure possesses a suitable band alignment, in which the VBM is lower than the oxidation potential and the CBM is higher than the reduction potential. Conversely, as shown in Figure S11, Supporting Information, when the biaxial strain is $-10 \%,-8 \%,+4 \%$, and $+6 \%$, the CBM and VBM are not simultaneously consistent with the suitable redox potential of water. To elaborate the relationship between energy alignment and biaxial strain, the statistical data were obtained from the CBM on the bottom side and VBM on the top side of $\mathrm{GeS} / \mathrm{MoSe}_{2}$ heterostructure via various biaxial strains. As shown in Figure $5 d$, the $\mathrm{GeS} / \mathrm{MoSe}_{2}$ heterostructure possesses a suitable band alignment and possible applications in the photocatalytic water splitting in the strain range from -7 to $+3 \%$.

In summary, we propose a 2D polarized $\mathrm{GeS} / \mathrm{MoSe}_{2}$ vdW heterostructure for water splitting using full sunlight. The structural stability, binding energy, internal electric field, optoelectronic properties, and photocatalytic activity of the $\mathrm{GeS} / \mathrm{MoSe}_{2}$ heterostructure were systematically investigated via first principles calculations. The results indicate that the advantageous properties of each material are well preserved in the GeS/MoSe 2 vdW heterostructure. The performance of $\mathrm{GeS} / \mathrm{MoSe}_{2}$ heterostructure 

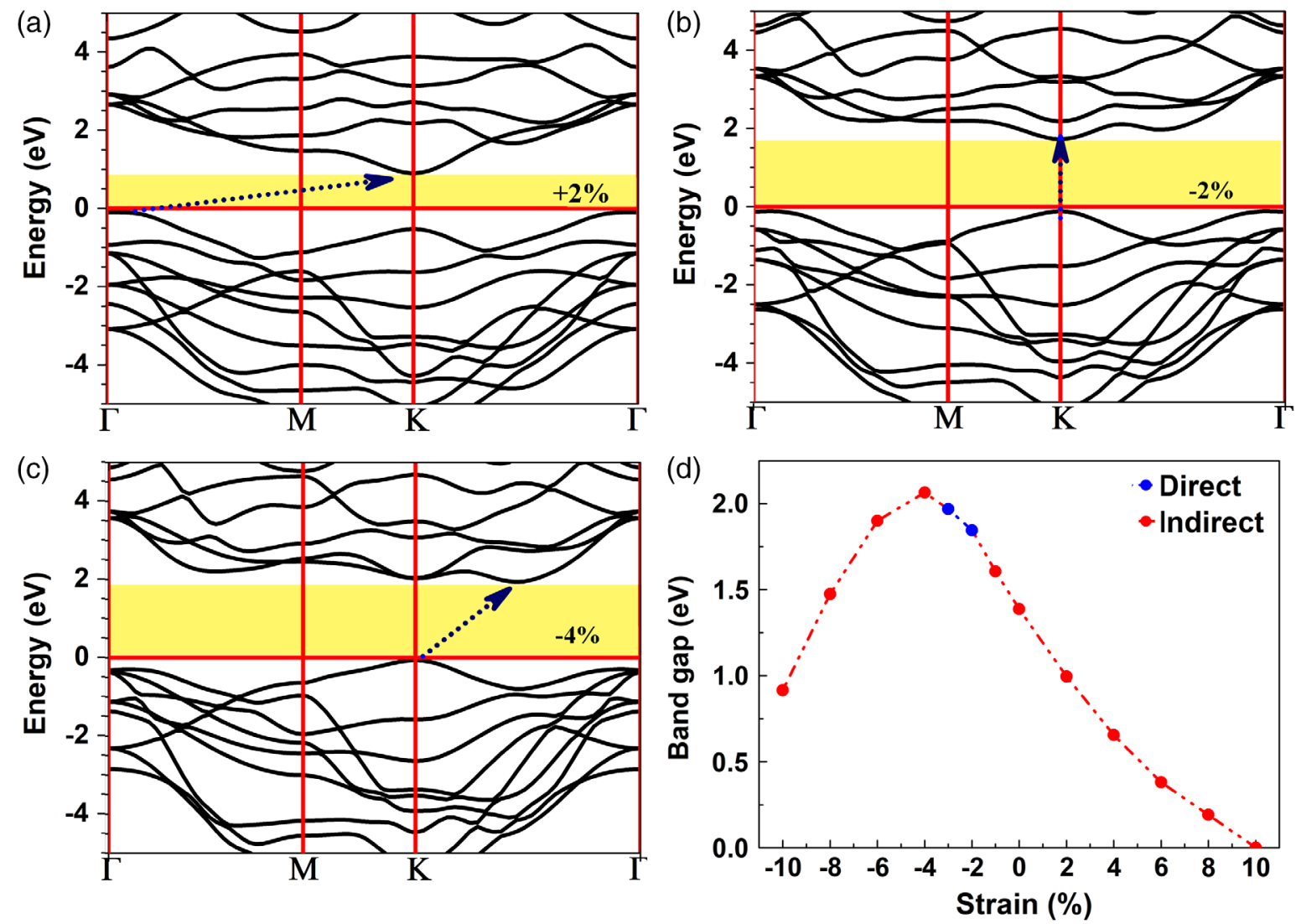

Figure 4. The band structures (HSE06) of GeS/MoSe 2 heterostructure at a) $+2 \%$, b) $-2 \%$, and c) $-4 \%$ biaxial strain, respectively. d) The bandgap as a function of biaxial strain of $\mathrm{GeS} / \mathrm{MoSe}_{2}$ heterostructure.

is better than isolated materials, as the properties of GeS monolayer and $\mathrm{MoSe}_{2}$ monolayer are complementary by forming vdW heterostructure, such as, the bandgap of the GeS/ $\mathrm{MoSe}_{2}$ heterostructure is about $1.387 \mathrm{eV}$, which is helpful to harvest a broader light spectrum range from UV to visible light; comparing with that only UV light is harvested by the isolated $\mathrm{GeS}$ monolayer. The GeS/MoSe 2 heterostructure possesses a similar dipole-induced internal electric field as that in GeS, where the direction pointing in the dipole-induced internal electric field is from the bottom surface to the top surface. Such an internal electric field reduces the probability of recombination of the photogenerated electron-hole pairs effectively and the photocatalyst's bandgap requirement for water splitting. Furthermore, the GeS/MoSe 2 heterostructure possesses excellent solar absorption performance, which is contributed mainly by the isolated $\mathrm{MoSe}_{2}$ monolayer. A wide light spectrum ranging from UV to near-IR light can be utilized with a high absorption coefficient $\left(10^{6} \mathrm{~cm}^{-1}\right)$, which greatly enhanced the solar energy conversion efficiency for water splitting. The band alignments of the GeS/MoSe 2 heterostructure well satisfy the redox potential required for the water-splitting reaction, indicating a harmonious ability to produce hydrogen and oxygen. In addition, it is feasible to tune the electronic and optical properties and enhance the photocatalytic activity of $\mathrm{GeS} / \mathrm{MoSe}_{2}$ heterostructure via strain engineering. The biaxial compressive strain range from $-2 \%$ to $-3 \%$ induces the direct bandgap character in $\mathrm{GeS} / \mathrm{MoSe}_{2}$ heterostructure. With a suitable bandgap, dipole-induced internal electric field, excellent solar absorption performance, and suitable band alignments, 2D polarized material-based $\mathrm{GeS} / \mathrm{MoSe}_{2} \mathrm{vdW}$ heterostructure is a potential novel high-efficiency photocatalyst for water splitting under a wide range of spectra from UV to near-IR light.

Vienna ab initio simulation package (VASP) in conjunction with the projector-augmented-wave (PAW) potential was used in all the calculations. ${ }^{[65,66]}$ The exchange-correlation energy was described by the Perdew-Burke-Ernzerhof (PBE) functional of the generalized gradient approximation (GGA). To obtain a more accurate bandgap, the HSE06 hybrid functional ${ }^{[67]}$ was used. The vdW density functional of optB88 ${ }^{[68-70]}$ was considered to describe long-range $\mathrm{vdW}$ interactions to give an improved description for GeS/MoSe 2 vdW heterostructure. The energy cutoff for the plane-wave expansion of the wave function was set to $500 \mathrm{eV}$. All the structures were fully relaxed until satisfying an energy convergence of $10^{-6} \mathrm{eV}$ and the maximum Hellmann Feynman force convergence of $0.001 \mathrm{eV} \AA^{-1}$. The gamma center scheme was used for the first Brillouin zone integration ${ }^{[71]}$ with a fine grid of $8 \times 8 \times 1$ and $11 \times 11 \times 1$ for structure optimization and static calculation, respectively. The thickness of the vacuum region along the $z$ direction was more than $20 \AA$ to separate the artificial interactions due to the periodic image. 

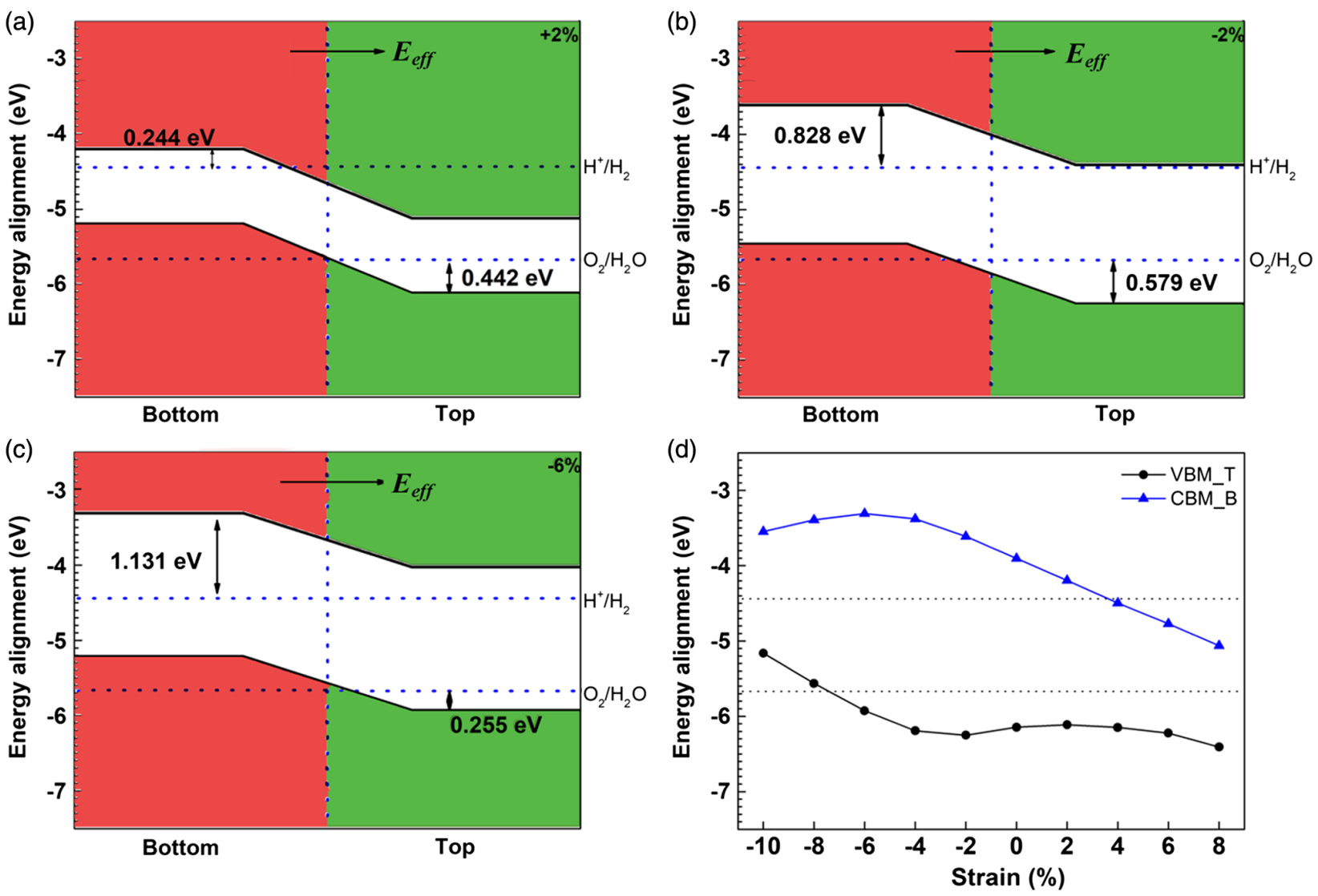

Figure 5. The energy alignment of GeS/MoSe heterostructure at a) $+2 \%, b)-2 \%$, and c) $-6 \%$ biaxial strain, respectively. d) The energy alignment as a function of biaxial strain of $\mathrm{GeS} / \mathrm{MoSe}_{2}$ heterostructure.

\section{Supporting Information}

Supporting Information is available from the Wiley Online Library or from the author.

\section{Acknowledgements}

The authors acknowledge the financial support from National Natural Science Foundation of China (11464001).

\section{Author Contributions}

D.G., Y.O., and Q.P. designed the project. D.G., X.T., H.C., and W.Z. conducted the simulations. D.G., X.T., Y.D., and Y.O. performed the analysis. D.C., Y.O., and Q.P. wrote the article. All authors discussed and commented on the manuscript.

\section{Conflict of Interest}

The authors declare no conflict of interest.

\section{Keywords}

$\mathrm{GeS} / \mathrm{MoSe}_{2}$, polarized materials, van der Waals heterostructures, water splitting
Received: October 7, 2019 Revised: October 30, 2019 Published online: November 14, 2019

[1] A. J. Esswein, D. G. Nocera, Chem. Rev. 2007, 107, 4022.

[2] W. Lubitz, W. Tumas, Chem. Rev. 2007, 107, 3900.

[3] J. Liu, Y. Liu, N. Liu, Y. Han, X. Zhang, H. Huang, Y. Lifshitz, S. T. Lee, J. Zhong, Z. Kang, Science 2015, 347, 970.

[4] W. Hu, J. Yang, J. Mater. Chem. C 2017, 5, 12289.

[5] C. F. Fu, X. Wu, J. Yang, Adv. Mater. 2018, 30, 1802106.

[6] A. Fujishima, K. Honda, Nature 1972, 238, 37.

[7] R. Asahi, T. Morikawa, T. Ohwaki, K. Aoki, Y. Taga, Science 2001, 293, 269.

[8] W. Choi, A. Termin, M. R. Hoffmann, J. Phys. Chem. 1994, 98, 13669.

[9] U. Diebold, Surf. Sci. Rep. 2003, 48, 53.

[10] H. G. Yang, C. H. Sun, S. Z. Qiao, J. Zou, G. Liu, S. C. Smith, H. M. Cheng, G. Q. Lu, Nature 2008, 453, 638.

[11] K. M. Lee, C. W. Lai, K. S. Ngai, J. C. Juan, Water Res. 2016, 88, 428.

[12] N. Daneshvar, D. Salari, A. R. Khataee, J. Photochem. Photobiol. A 2004, 162, 317.

[13] M. Matsumura, S. Furukawa, Y. Saho, H. Tsubomura, J. Phys. Chem. 1985, 89, 1327.

[14] X. Zong, H. Yan, G. Wu, G. Ma, F. Wen, L. Wang, C. Li, J. Am. Chem. Soc. 2008, 130, 7176.

[15] K. Zhang, L. Guo, Catal. Sci. Technol. 2013, 3, 1672.

[16] K. Domen, A. Kudo, T. Onishi, J. Catal. 1986, 102, 92. 
[17] K. Domen, S. Naito, T. Onishi, K. Tamaru, Chem. Phys. Lett. 1982, 92, 433.

[18] T. Su, Q. Shao, Z. Qin, Z. Guo, Z. Wu, ACS Catal. 2018, 8, 2253.

[19] X. Jiang, P. Wang, J. Zhao, J. Mater. Chem. A 2015, 3, 7750.

[20] Y. Jiao, L. Zhou, F. Ma, G. Gao, L. Kou, J. Bell, S. Sanvito, A. Du, ACS Appl. Mater. Interfaces 2016, 8, 5385.

[21] H. L. Zhuang, R. G. Hennig, Chem. Mater. 2013, 25, 3232.

[22] Y. Sang, Z. Zhao, M. Zhao, P. Hao, Y. Leng, H. Liu, Adv. Mater. 2015, 27, 363 .

[23] C. Fu, X. Li, Q. Luo, J. Yang, J. Mater. Chem. A 2017, 5, 24972.

[24] C. Xia, W. Xiong, J. Du, T. Wang, Y. Peng, J. Li, Phys. Rev. B 2018, 98, 165424.

[25] Y. Ji, M. Yang, H. Lin, T. Hou, L. Wang, Y. Li, S.-T. Lee, J. Phys. Chem. C 2018, 122, 3123.

[26] Y. Ji, M. Yang, H. Dong, T. Hou, L. Wang, Y. Li, Nanoscale 2017, 9, 8608.

[27] X. Li, Z. Li, J. Yang, Phys. Rev. Lett. 2014, 112, 018301.

[28] C. Fu, J. Sun, Q. Luo, X. Li, W. Hu, J. Yang, Nano Lett. 2018, 18, 6312.

[29] Y. Li, J. Wang, B. Zhou, F. Wang, Y. Miao, J. Wei, B. Zhang, K. Zhang, Phys. Chem. Chem. Phys. 2018, 20, 24109.

[30] Y. Liang, J. Li, H. Jin, B. Huang, Y. Dai, J. Phys. Chem. Lett. 2018, 9, 2797.

[31] X. Ma, X. Wu, H. Wang, Y. Wang, J. Mater. Chem. A 2018, 6, 2295.

[32] D. Gu, X. Tao, H. Chen, W. Zhu, Y. Ouyang, Q. Peng, Nanoscale 2019, 11,2335

[33] A. K. Geim, I. V. Grigorieva, Nature 2013, 499, 419.

[34] Y. Deng, Z. Luo, N. J. Conrad, H. Liu, Y. Gong, S. Najmaei, P. M. Ajayan, J. Lou, X. Xu, P. D. Ye, ACS Nano 2014, 8, 8292.

[35] V. D. S. O. Ganesan, J. Linghu, C. Zhang, Y. P. Feng, S. Lei, Appl. Phys. Lett. 2016, 108, 122105.

[36] V. O. Ozcelik, J. G. Azadani, C. Yang, S. J. Koester, T. Low, Phys. Rev. B 2016, 94, 035125.

[37] L. Ju, Y. Dai, W. Wei, M. Li, B. Huang, Appl. Surf. Sci. 2018, 434, 365.

[38] B. Wang, A. Kuang, X. Luo, G. Wang, H. Yuan, H. Chen, Appl. Surf. Sci. 2018, 439, 374

[39] S. Wang, C. Ren, H. Tian, J. Yu, M. Sun, Phys. Chem. Chem. Phys. 2018, 20, 13394.

[40] Z. Zhang, Q. Qian, B. Li, K. J. Chen, ACS Appl. Mater. Interfaces 2018, 10, 17419.

[41] Y. Yuan, Z. Shen, S. Wu, Y. Su, L. Pei, Z. Ji, M. Ding, W. Bai, Y. Chen, Z. Yu, Z. Zou, Appl. Catal. B 2019, 246, 120.

[42] C. F. Fu, R. Zhang, Q. Luo, X. Li, J. Yang, J. Comput. Chem. 2019, 40, 980.

[43] B. Wang, X. Li, X. Cai, W. Yu, L. Zhang, R. Zhao, S. Ke, J. Phys. Chem. C 2018, 122, 7075.
[44] Y. Qun, T. Chunjian, M. Ruishen, J. Junke, L. Qiuhua, S. Xiang Y. Daoguo, C. Xianping, IEEE Electron Device Lett. 2017, 38, 145.

[45] B. Wang, X. Li, R. Zhao, X. Cai, W. Yu, W. Li, Z. Liu, L. Zhang, S. Ke, J. Mater. Chem. A 2018, 6, 8923.

[46] R. Zhang, L. Zhang, Q. Zheng, P. Gao, J. Zhao, J. Yang, J. Phys. Chem. Lett. 2018, 9, 5419 .

[47] X. She, J. Wu, H. Xu, J. Zhong, Y. Wang, Y. Song, K. Nie, Y. Liu Y. Yang, M. T. F. Rodrigues, Adv. Energy Mater. 2017, 7, 1700025.

[48] Y. Li, Y. Li, B. Sa, R. Ahuja, Catal. Sci. Technol. 2017, 7, 545.

[49] P. Kumar, R. Boukherroub, K. Shankar, J. Mater. Chem. A 2018, 6, 12876.

[50] T. Hu, J. Dong, Phys. Chem. Chem. Phys. 2016, 18, 32514.

[51] C. Chang, X. Fan, S. Lin, J. Kuo, Phys. Rev. B 2013, 88, 195420.

[52] Y. Ding, Y. Wang, J. Ni, L. Shi, S. Shi, W. Tang, Physica B 2011, 406,2254

[53] S. Wei, F. Wang, P. Yan, M. Dan, W. Cen, S. Yu, Y. Zhou, J. Catal. 2019, 377, 122.

[54] Y. Lin, H. Shi, Z. Jiang, G. Wang, X. Zhang, H. Zhu, R. Zhang, C. Zhu, Int. J. Hydrogen Energy 2017, 42, 9903.

[55] L. Peng, Y. Cui, L. Sun, J. Du, S. Wang, S. Zhang, Y. Huang, Nanoscale Horiz. 2019, 4, 480

[56] C. Xia, J. Du, W. Xiong, Y. Jia, Z. Wei, J. Li, J. Mater. Chem. A 2017, 5, 13400.

[57] Q. Peng, Z. Wang, B. Sa, B. Wu, Z. Sun, Sci. Rep. 2016, 6, 31994.

[58] W. Zhang, L. Zhang, RSC Adv. 2017, 7, 34584.

[59] C. Xia, J. Du, X. Huang, W. Xiao, W. Xiong, T. Wang, Z. Wei, Y. Jia, J. Shi, J. Li, Phys. Rev. B 2018, 97, 115416.

[60] D. Gu, X. Tao, H. Chen, Y. Ouyang, W. Zhu, Q. Peng, Y. Du, Phys. Status Solidi RRL 2019, 13, 1800659.

[61] J. Feng, X. Qian, C.-W. Huang, J. Li, Nat. Photonics 2012, 6, 866.

[62] N. Lu, H. Guo, L. Li, J. Dai, L. Wang, W.-N. Mei, X. Wu, X. C. Zeng, Nanoscale 2014, 6, 2879.

[63] Y. He, Y. Yang, Z. Zhang, Y. Gong, W. Zhou, Z. Hu, G. Ye, X. Zhang E. Bianco, S. Lei, Z. Jin, X. Zou, Y. Yang, Y. Zhang, E. Xie, J. Lou, B. Yakobson, R. Vajtai, B. Li, P. Ajayan, Nano Lett. 2016, 16, 3314.

[64] Z. Fan, X. Jiang, Z. Wei, J. Luo, S. Li, J. Phys. Chem. C 2017, 121, 14373.

[65] G. Kresse, J. Furthmüller, Phys. Rev. B 1996, 54, 11169.

[66] P. E. Blochl, Phys. Rev. B 1994, 50, 17953.

[67] J. Paier, M. Marsman, K. Hummer, G. Kresse, I. C. Gerber, J. G. Angyan, J. Chem. Phys. 2006, 124, 154709.

[68] A. D. Becke, Phys. Rev. A 1988, 38, 3098.

[69] J. Klimeš, D. R. Bowler, A. Michaelides, J. Phys.: Condens. Matter 2009, 22, 022201.

[70] J. Klimeš, D. R. Bowler, A. Michaelides, Phys. Rev. B 2011, 83, 195131.

[71] H. J. Monkhorst, J. D. Pack, Phys. Rev. B 1976, 13, 5188. 